\title{
The era after DAWN: treatment of acute ischaemic stroke
}

\author{
KK Lau ${ }^{1 *}$, FHKAM (Medicine), FRCP, Thomas WH Leung ${ }^{2}$, FRCP, FHKAM (Medicine), \\ Yannie OY Soo², MRCP, FHKAM (Medicine) \\ ${ }^{1}$ Department of Medicine and Geriatrics, Princess Margaret Hospital, Laichikok, Hong Kong \\ ${ }^{2}$ Department of Medicine and Therapeutics, Prince of Wales Hospital, The Chinese University of Hong Kong, Shatin, Hong Kong \\ Hong Kong Med J 2018;24:313-5 \\ *Corresponding author: laukk2@ha.org.hk \\ DOI: 10.12809/hkmj176912
}

Intravenous tissue plasminogen activator (TPA) is the only proven medical treatment for acute ischaemic stroke. ${ }^{1}$ Early recanalisation of occluded vessels can be achieved in $30 \%$ to $46 \%$ of cases. ${ }^{1}$ Prognosis for patients who fail recanalisation is mostly dismal unless endovascular thrombectomy can be performed in a timely manner. ${ }^{2}$ Over the last few years, neurologists around the world have witnessed a paradigm shift in treating stroke patients with moderate-to-severe neurological disability (ie, an National Institute of Health Stroke Scale score of 10-25, or even higher). ${ }^{3}$

Five randomised studies that recruited patients refractory to TPA (MR CLEAN, ESCAPE, REVASCAT, SWIFT PRIME, and EXTEND IA) showed that endovascular thrombectomy improved clinical outcomes in terms of reducing functional disability at 90 days (ie, a modified Rankin Scale [mRS] of $0-2){ }^{3}$ In a meta-analysis of these five studies by HERMES collaboration, the investigators directly accessed and extracted patient data at an individual level. They used mixed-effects modelling with random effects for parameters of interest, and mixed-effects ordinal logistic regression models to calculate common odds ratios (cOR). In both cases, they adjusted the age, gender, baseline stroke severity, site of occlusion, baseline Alberta Stroke Program Early CT Score, time from onset to randomisation, and whole population (shift analysis). ${ }^{3}$ Of 1287 patients studied, 634 underwent endovascular thrombectomy and 653 received TPA. Those who underwent endovascular thrombectomy had reduced disability, with $\mathrm{mRS}$ of 0 to 2 at 90 days, and an adjusted cOR of 2.49 (95\% confidence interval $[\mathrm{CI}]=1.76-3.53 ; \quad \mathrm{P}<0.0001)$. The number-neededto-treat to reduce one level of disability on mRS was 2.6. There was no heterogeneity of treatment effect across all subgroups, including patients older than 80 years (cOR 3.68, 95\% CI=1.95-6.92) and randomisation more than 300 minutes $(1.76,1.05-$ 2.97). ${ }^{3}$ Among the five trials, only MR CLEAN had complete recruitment ${ }^{4}$; the other four studies were prematurely terminated due to the high probability of improvement according to interim analyses. ${ }^{3}$

Although endovascular thrombectomy studies have reset the treatment paradigm for large cerebral vessel occlusion stroke, the recently published DAWN (DWI or CTP Assessment with Clinical Mismatch in the Triage of Wake-Up and Late Presenting Strokes Undergoing Neurointervention with Trevo) study went one step further in this revolution. ${ }^{5,6}$

The DAWN trial extended the therapeutic time window for endovascular thrombectomy to up to 24 hours in carefully selected patients based on a clinical-radiological mismatch. ${ }^{5}$ The study enrolled patients with acute large intracranial vessel occlusion, namely the terminal internal carotid artery or M1 middle cerebral artery (on computed tomography [CT] or magnetic resonance angiography), who presented after 6 to 24 hours, with a mismatch between the severity of clinical deficit and infarct volume (measured by magnetic resonance diffusion-weighted imaging or perfusion $\mathrm{CT}$ ) and defined according to age ( $<80$ years or $\geq 80$ years). Patients were randomised to receive standard care plus endovascular thrombectomy or standard care alone. The two end points were mean score for disability on the utility-weighted $\mathrm{mRS}$, ranging from 0 (death) to 10 (no symptoms or disability) and functional independence, or mRS of 0 to 2 at 90 days. ${ }^{6}$ The DAWN study was prematurely stopped since the prespecified interim analysis showed a high probability of superior outcome in the thrombectomy group. ${ }^{6}$ Among the 206 patients recruited, 107 were in the thrombectomy group and 99 were in the control group. At 31 months, the mean score of utility-weighted mRS at 90 days was 5.5 in the thrombectomy group and 3.4 in the control group; adjusted difference (Bayesian analysis) was 2.0 points, with $95 \%$ credible interval of 1.1 to 3.0 and posterior probability of superiority being $>0.999$. The adjusted functional independence at 90 days was $49 \%$ in the thrombectomy group and $13 \%$ in the control group ${ }^{6}$; adjusted difference was $33 \%$ points, with $95 \%$ credible interval of 24 to 44 and posterior probability of superiority being $>0.999$.

The salvageable tissue-based approach for patient selection was a key factor in DAWN. ${ }^{7}$ Despite the longer onset-to-treatment time compared with 
previous endovascular thrombectomy trials, the treatment benefit (number-needed-to-treat=2 for better score for disability at 90 days) remains highest among all the acute stroke trials to date. The decision for reperfusion therapy in acute stroke has been conventionally governed by a definitive 'time window' Replacing clock time by salvageable tissue provided treatment opportunities for $60 \%$ of patients in this trial who awake with a stroke, and $14.3 \%$ of all ischaemic stroke patients who presented to the emergency department. ${ }^{8}$

The benefit of early intravenous TPA has been proven for decades. ${ }^{1}$ In the real world, due to various limitations, its availability varies widely among countries. ${ }^{9,10}$ According to the Management Information Portal (an intranet for the Hospital Authority staff), in 2015-2016 there were about 11000 patients with acute ischaemic stroke and unspecified stroke in Hong Kong. With around 70 neurologists in the public sector serving a population of more than 7 million, the narrow therapeutic window for reperfusion therapy poses a great challenge to an acute stroke service. Since 2012, the introduction of telestroke with iPad has expedited the development of 24-hour thrombolytic therapy in Hong Kong. Through videoconferencing and teleradiology, off-site neurologists can now promptly evaluate acute stroke patients for thrombolytic therapy despite the absence or inadequacy of on-site specialist support. Nonetheless, although advances in technology can overcome geographical barriers and bring expertise to the bedside, it will not replace the need for stroke specialists who make treatment decisions through teleconsultation. Currently, a round-the-clock 24/7 TPA service for acute stroke is available in only seven of the 17 acute hospitals in Hong Kong. This huge service gap calls for an immediate input of resources for team development (including neurologists, emergency physicians, radiologists, radiographers, and stroke nurses) and expansion of imaging facilities to allow a uniform service provision to all people in Hong Kong.

Despite the extended therapeutic time window in the DAWN trial, treatment should not be delayed because as many as two million neurons die every minute in an ischaemic brain. ${ }^{11}$ Every effort should be made to shorten the door-to-treatment time, recommended to be less than 60 minutes. ${ }^{12}$ An optimal TPA protocol could reduce a median doorto-needle time from 61 to 46 minutes. ${ }^{13}$ The Angels programme is a global initiative that aims to optimise quality of acute stroke treatment. ${ }^{14}$ Participating centres are invited to compare their performance with that recommended by international guidelines. A gap analysis can be conducted to identify areas for improvement. The Angels initiative offers online training to members of the stroke teams with the aim of reducing onset-to-door time and door-to- needle time. ${ }^{14}$

In conclusion, acute stroke therapy has advanced rapidly over the last few years. The extended therapeutic window for endovascular thrombectomy will substantially increase the number of stroke patients who require urgent assessment, timely neuroimaging tests, and immediate endovascular intervention.

Although the Hospital Authority should take the lead and proactively improve the acute stroke care system, health care providers should also prepare themselves for the surging awareness of stroke and treatment expectations of the general public after DAWN.

\section{Declaration}

As an editor of this journal, TWH Leung was not involved in the peer review process of this article. All other authors have no conflicts of interest to declare. All authors had full access to the data, contributed to the study, approved the final version for publication, and take responsibility for its accuracy and integrity.

\section{References}

1. National Institute of Neurological Disorders and Stroke rt-PA Stroke Study Group. Tissue plasminogen activator for acute ischemic stroke. N Engl J Med 1995;333:1581-7.

2. Heldner MR, Zubler C, Mattle HP, et al. National Institutes of Health stroke scale score and vessel occlusion in 2152 patients with acute ischemic stroke. Stroke 2013;44:11537.

3. Goyal M, Menon BK, van Zwam WH, et al. Endovascular thrombectomy after large-vessel ischaemic stroke: a metaanalysis of individual patient data from five randomised trials. Lancet 2016;387:1723-31.

4. Berkhemer OA, Fransen PS, Beumer D, et al. A randomized trial of intraarterial treatment for acute ischemic stroke. $\mathrm{N}$ Engl J Med 2015;372:11-20.

5. Bracard S, Ducrocq X, Mas JL, et al. Mechanical thrombectomy after intravenous alteplase versus alteplase alone after stroke (THRACE): a randomised controlled trial. Lancet Neurol 2016;15:1138-47.

6. Nogueira RG, Jadhav AP, Haussen DC, et al. Thrombectomy 6 to 24 hours after stroke with a mismatch between deficit and infarct. N Engl J Med 2018;378:11-21.

7. Dávalos A, Blanco M, Pedraza S, et al. The clinical-DWI mismatch: a new diagnostic approach to the brain tissue at risk of infarction. Neurology 2004;62:2187-92.

8. Mackey J, Kleindorfer D, Sucharew H, et al. Populationbased study of wake-up strokes. Neurology 2011;76:16627.

9. Hong KS, Saver JL. Quantifying the value of stroke disability outcomes: WHO global burden of disease project disability weights for each level of the modified rankin scale. Stroke 2009;40:3828-33.

10. King's College London for the Stroke Alliance for Europe (SAFE). The burden of stroke in Europe report. Available from: https://www.stroke.org.uk/sites/default/files/the burden_of_stroke_in_europe_-_challenges_for_policy_ makers.pdf. Accessed 11 Dec 2017.

11. Saver JL. Time is brain-quantified. Stroke 2006;37:263-6. 
12. Fonarow GC, Zhao X, Smith EE, et al. Door-to-needle times for tissue plasminogen activator administration and clinical outcomes in acute ischemic stroke before and after a quality improvement initiative. JAMA 2014;311:163240.
13. Meretoja A, Weir L, Ugalde M, et al. Helsinki model cut stroke thrombolysis delays to 25 minutes in Melbourne in only 4 months. Neurology 2013;81:1071-6.

14. Angels Community. Available from: https://angelsinitiative.com/. Accessed 14 Jul 2017. 